\title{
Synthesis and Characterization of Nanocomposite Films with a Titania Glass Matrix by the Sol-gel Route
}

\author{
T. K. Kundu and D. Chakravorty* $†$ \\ ${ }^{1}$ Indian Association for the Cultivation of Science, Jadavpur, Calcutta: 700 032, India \\ ${ }^{2}$ Also affiliated to Jawaharlal Nehru Centre for Advanced Scientific Research, Bangalore: 560 064, India
}

Films of thickness $\sim 3 \mu \mathrm{m}$ containing nanosized metal particles of iron, nickel and copper respectively were grown on a Corning glass slide using a sol-gel technique. The particle sizes had values in the range 6.8 to $20.1 \mathrm{~nm}$. Optical absorption characteristics of different specimens were studied over the wavelength range 230 to $830 \mathrm{~nm}$. An absorption maximum was observed at wavelength varying from 270 to $300 \mathrm{~nm}$ depending on the nanocomposite system. The data were analysed on the basis of effective medium theories viz., Maxwell-Garnett (MG) and Bruggemann (BR) models respectively. MG model showed better agreement with experimental results than BR theory. The volume fraction as estimated from the least square fitting procedure was found to be in the range 2 to 3\% . Copyright (C) 1999 John Wiley \& Sons, Ltd.

Keywords: thin films; nanocomposites; iron; nickel; copper; titania glass; optical absorption characteristics

\section{INTRODUCTION}

The Sol-gel technique has emerged as a powerful route to the synthesis of a wide range of materials. ${ }^{1}$ High purity and homogeneity can be attained by

* Correspondence to: D. Chakravorty, Indian Association for the Cultivation of Science, Jadavpur, Calcutta 700 032, India. E-mail: mls@iacs.ernet.in

$\dagger$ Also affiliated to Jawaharlal Nehru Centre for Advanced Scientific Research, Bangalore: 560 064, India.

Contract/grant sponsor: Office of Naval Research, Washington, DC, USA; Contract/grant number: N 00014-93-1-0040. this method which has the advantage of a low temperature processing requirement. ${ }^{2,3}$ A number of composites containing nanometre-sized metals dispersed in a silica glass matrix have been prepared using this method. ${ }^{4,5}$ These have been used to investigate the behaviour of materials in their nanocrystalline state. ${ }^{6-8}$ As part of a wider investigation we have explored the possibility of making nanocomposites with a titania $\left(\mathrm{TiO}_{2}\right)$ glass matrix. $\mathrm{TiO}_{2}$ cannot be made in the glassy form by the conventional melt-quench route. In the present work we have synthesized by the sol-gel method the nanocomposites containing iron, nickel and copper respectively dispersed in a $\mathrm{TiO}_{2}$-based glass matrix in thick-film form. Optical absorption characteristics of these films have been studied and the details are reported in this paper.

\section{EXPERIMENTAL}

A mixture of $120 \mathrm{~cm}^{3}$ of ethyl alcohol and $30 \mathrm{~cm}^{3}$ of acetic acid was stirred for $0.5 \mathrm{~h}$. After adding $18 \mathrm{~cm}^{3}$ of tetraisopropyl orthotitanate had been added to this mixture, it was stirred for $1 \mathrm{~h}$, the divided into three equal parts (denoted by $A_{1}, A_{2}$ and $A_{3}$ respectively). Three mixtures $\left(B_{1}, B_{2}\right.$ and $\mathrm{B}_{3}$ ) were then prepared:

$\left(\mathrm{B}_{1}\right) 1 \mathrm{~g}$ ferric chloride $+10 \mathrm{~cm}^{3}$ ethyl alcohol $+10 \mathrm{~cm}^{3}$ distilled water

$\left(\mathrm{B}_{2}\right) 2 \mathrm{~g}$ nickel chloride $+10 \mathrm{~cm}^{3}$ ethyl alcohol $+10 \mathrm{~cm}^{3}$ distilled water

$\left(\mathrm{B}_{3}\right) 2 \mathrm{~g}$ cupric chloride $+10 \mathrm{~cm}^{3}$ ethyl alcohol $+10 \mathrm{~cm}^{3}$ distilled water

These are denoted by $\mathrm{B}_{1}, \mathrm{~B}_{2}$ and $\mathrm{B}_{3}$ respectively. Finally three sols were prepared by mixing $A_{1}$ and $\mathrm{B}_{1}, \mathrm{~A}_{2}$ and $\mathrm{B}_{2}, \mathrm{~A}_{3}$ and $\mathrm{B}_{3}$ respectively.

Films were prepared by dipping chemically cleaned Corning glass slides (dimensions $3 \mathrm{~cm}$ $\times 1 \mathrm{~cm} \times 0.1 \mathrm{~cm}$ into the solutions prepared as 

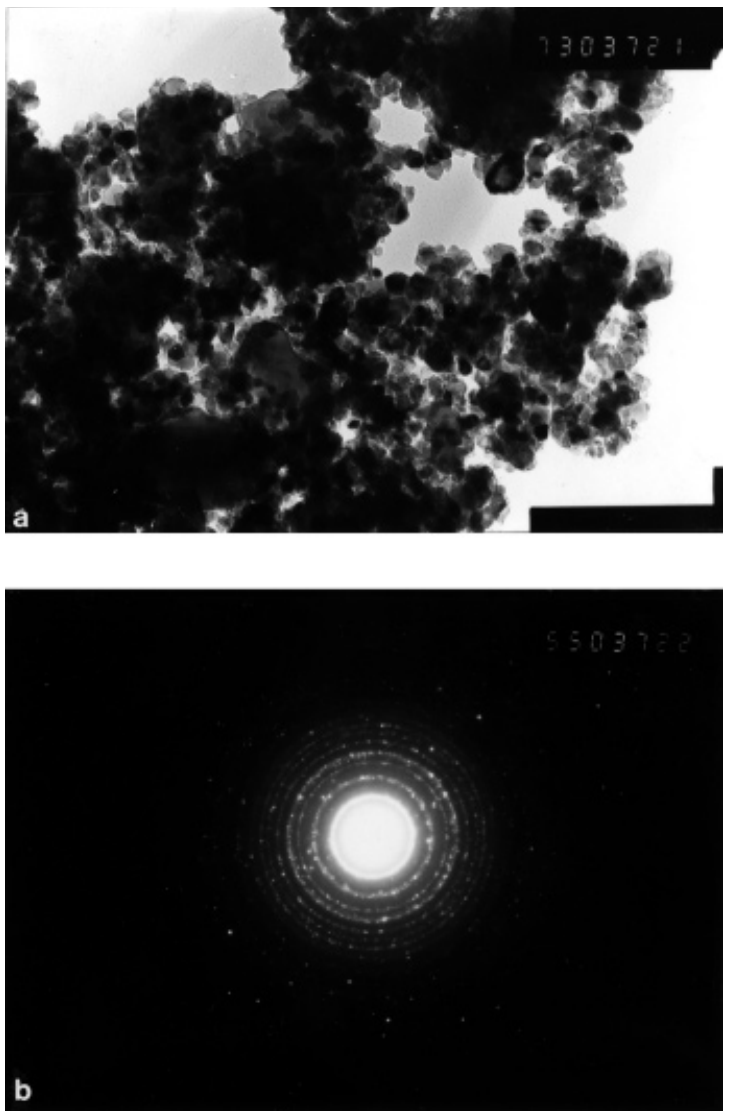

Figure 1 (a) Transmission electron micrograph of specimen containing iron and reduced at $675^{\circ} \mathrm{C}$ for $20 \mathrm{~min}$; (b) electron diffraction pattern of the same specimen.

described above. The films were initially dried at room temperature, then oven dried at $100^{\circ} \mathrm{C}$ for $24 \mathrm{~h}$, heated slowly to $300^{\circ} \mathrm{C}$ and kept for $1 \mathrm{~h}$. Finally they were subjected to reduction treatment in hydrogen at temperatures in the range 550 to $675^{\circ} \mathrm{C}$ for $10-20$ minutes to precipitate nanosized metal particles within the gel-derived glass matrix. The thicknesses of the films were measured by an SF 220 surface profiler supplied by Planer Products Ltd., U.K. The films were found to have thicknesses in the range 3 to $4 \mu \mathrm{m}$.

For microstructural studies gel pieces having compositions identical to those described as above and subjected to the same reduction treatment were finely ground in an agate mortar. The resulting powders were dispersed in acetone kept in a test tube which was then placed in an ultrasonic bath for about $0.5 \mathrm{~h}$. A drop of this dispersion was then deposited on a carbon coated copper grid which was dried in air and subsequently mounted in the sample
Table 1. Comparison of interplanar spacings $d_{h k l} \mathrm{~nm}$ obtained from electron diffraction data with ASTM values for $\mathrm{Fe} / \mathrm{TiO}_{2}$ specimen reduced at $675^{\circ} \mathrm{C}$ for $20 \mathrm{~min}$

\begin{tabular}{lll}
\hline & \multicolumn{2}{c}{$\mathrm{A} \mathrm{S} \mathrm{T} \mathrm{M}$} \\
\cline { 2 - 3 } Observed & $\mathrm{Fe}$ & $\mathrm{TiO}_{2}$ \\
\hline 0.271 & & 0.252 \\
0.219 & & 0.218 \\
0.202 & 0.2026 & 0.205 \\
0.178 & & \\
0.1587 & & 0.1624 \\
0.148 & & 0.148 \\
0.141 & 0.143 & 0.145 \\
0.123 & & 0.120 \\
\hline
\end{tabular}

chamber of a JEM 200 CX transmission electron microscope.

The optical absorption characteristics of the nanocomposite films were determined by taking the spectra in a Hitachi 300 Spectrophotometer over the wavelength range 230 to $830 \mathrm{~nm}$. The absorption coefficient was calculated from equation [1]:

$$
\alpha=\frac{1}{t} \ln \left(\frac{1}{T}\right)
$$

where $\mathrm{T}$ is the transmittance of the sample and $\mathrm{t}$ its thickness.

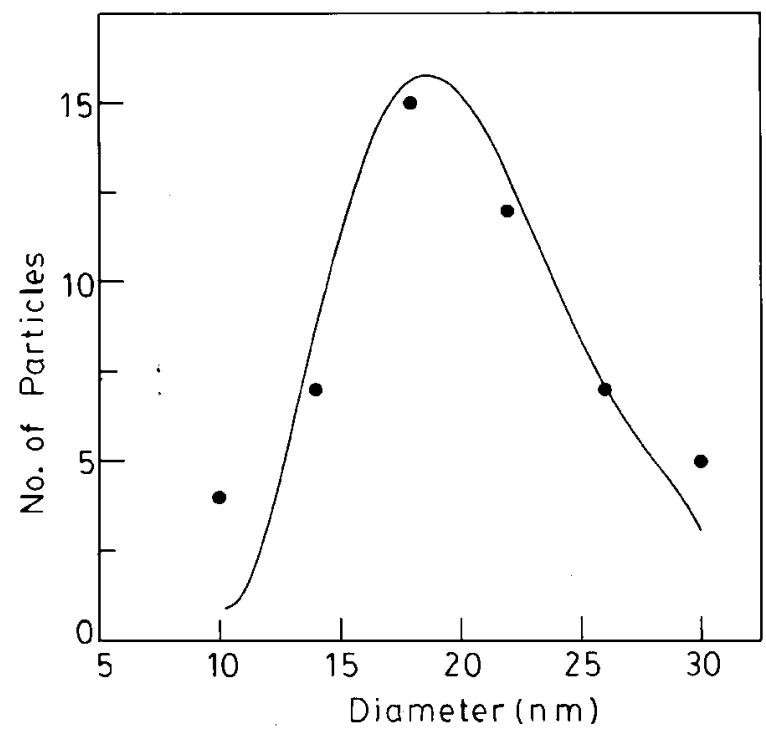

Figure 2 Particle size distribution of the specimen shown in Fig. 1: experimental; —, theoretical.

Appl. Organometal. Chem. 13, 353-360 (1999) 

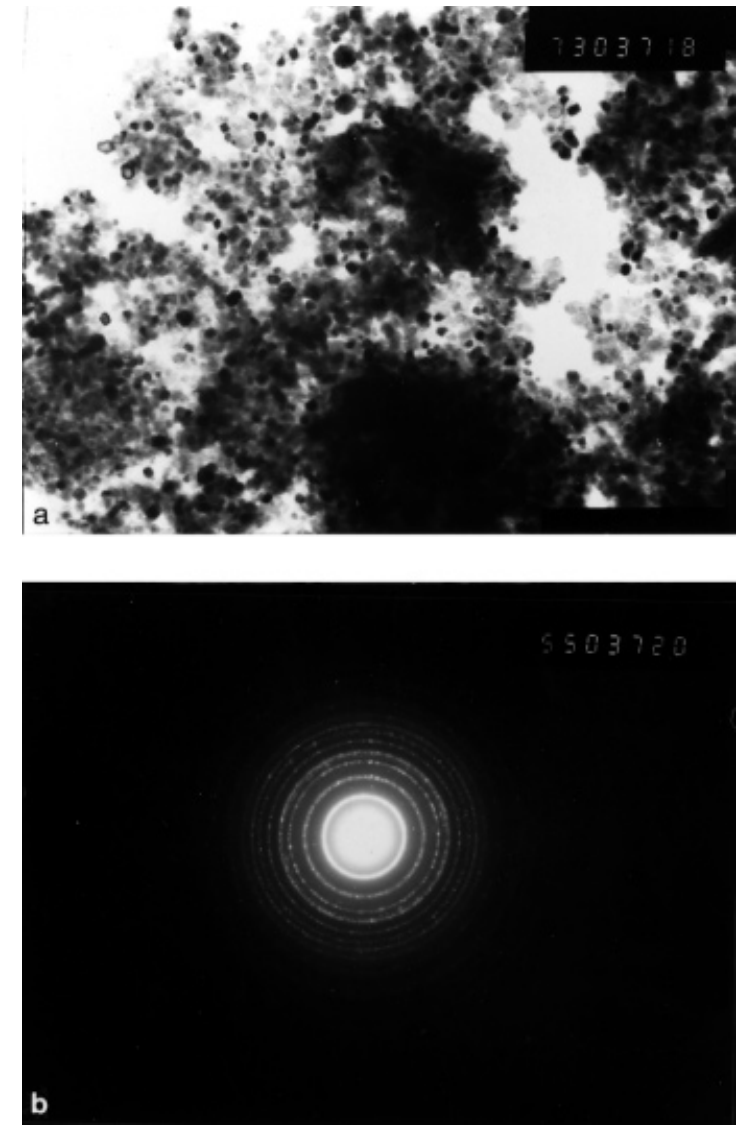

Figure 3 (a) Transmission electron micrograph of specimen containing nickel and reduced at $660^{\circ} \mathrm{C}$ for $10 \mathrm{~min}$; (b) electron diffraction pattern of the same specimen.

\section{RESULTS AND DISCUSSION}

Figure 1(a) shows the transmission electron micrograph for a specimen containing iron which was reduced at $675^{\circ} \mathrm{C}$ for 20 minutes. Figure 1(b) is the electron diffraction pattern. Interplanar spacing $\left(d_{h k 1}\right)$ values have been calculated from the diameters of the rings and are summarised in Table 1. Standard $d_{h k l}$ values for metallic iron as well as titania are also listed in the table. It is evident from the observed values that both iron and titania particles are present in this sample. The microstructure shows a distribution of particles having diameters in the range 10 to $30 \mathrm{~nm}$. The data have been fitted to a log normal distribution function given by equation [2].

$$
\Delta n=\frac{1}{\sqrt{2 \pi} \ln \sigma} \exp \left\{-\left[\ln \left(\frac{x}{\bar{x}}\right) / \ln \sigma\right]\right\} \Delta(\ln x)
$$
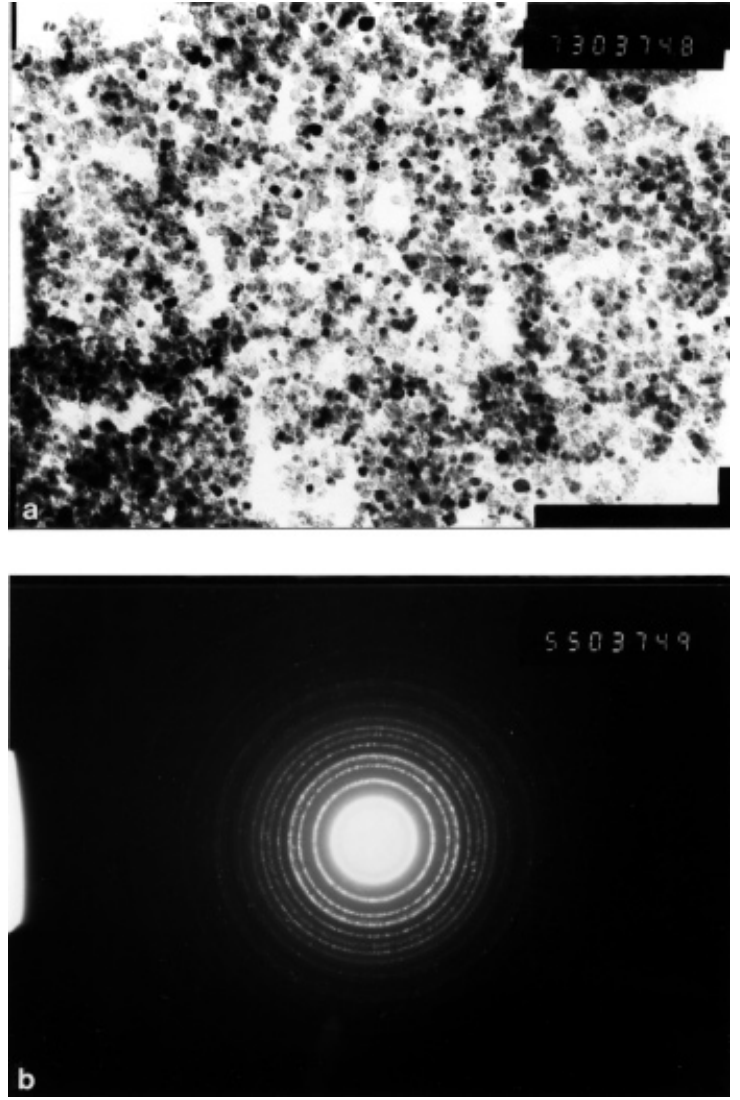

Figure 4 (a) Transmission electron micrograph of specimen containing copper and reduced at $660{ }^{\circ} \mathrm{C}$ for $10 \mathrm{~min}$; (b) electron diffraction pattern of the same specimen.

where $\Delta n$ is the fractional number of particles per logarithmic diameter interval $\Delta(\ln x), \bar{x}$ is the median diameter and $\sigma$ the geometric standard deviation. The particle size distribution is shown in Fig. 2, in which the points represent the experimental data and the solid line the least-squares fitted curve using Equation [2]. The extracted values of $\bar{x}$ and $\sigma$ are shown in Table 4 (below). The microstructure and the electron diffraction rings as shown in Fig. 1 are typical of all other samples containing $\mathrm{Fe}$ and $\mathrm{TiO}_{2}$ particles.

The electron micrograph and electron diffraction pattern of a specimen containing nickel and subjected to a reduction treatment at $660^{\circ} \mathrm{C}$ for 10 min are shown in Figs 3(a) and 3(b) respectively. Figures 4(a) and 4(b) show the micrograph and electron diffraction pattern for a specimen containing copper which was subjected to a reduction treatment at $660{ }^{\circ} \mathrm{C}$ for $10 \mathrm{~min}$. These are typical of 
Table 2. Comparison of $\boldsymbol{d}_{h k l} \mathrm{~nm}$ obtained from electron diffraction data with ASTM values for $\mathrm{Ni} / \mathrm{TiO}_{2}$ specimen reduced at $660^{\circ} \mathrm{C}$ for $10 \mathrm{~min}$

\begin{tabular}{lcl}
\hline Observed & $\begin{array}{c}\text { ASTM } \\
\mathrm{Ni}\end{array}$ & $\mathrm{TiO}_{2}$ \\
\hline 0.271 & & 0.252 \\
0.219 & & 0.218 \\
0.202 & 0.2034 & 0.205 \\
0.178 & 0.1762 & \\
0.1587 & & 0.1624 \\
0.148 & & 0.148 \\
0.141 & & 0.145 \\
0.123 & 0.1246 & 0.120 \\
\hline
\end{tabular}

Table 3. Comparison of $d_{h k l} \mathrm{~nm}$ obtained from electron diffraction data with ASTM values for $\mathrm{Cu} / \mathrm{TiO}_{2}$ specimen: reduced at $660^{\circ} \mathrm{C}$ for $10 \mathrm{~min}$

\begin{tabular}{lll}
\hline Observed & $\begin{array}{c}\mathrm{ASTM} \\
\mathrm{Cu}\end{array}$ & $\mathrm{TiO}_{2}$ \\
\hline 0.271 & & 0.252 \\
0.219 & & 0.218 \\
0.208 & 0.2088 & 0.205 \\
0.202 & & \\
0.178 & 0.180 & \\
0.1587 & & 0.1624 \\
0.148 & & 0.148 \\
0.141 & & 0.145 \\
0.123 & 0.1278 & 0.120 \\
\hline
\end{tabular}

all specimens in these two series. Tables 2 and 3 show the calculated values of $d_{h k l}$ as obtained from Figs 3(b) and 4(b) respectively. Comparison with ASTM values shows the presence of $\mathrm{TiO}_{2}$ particles

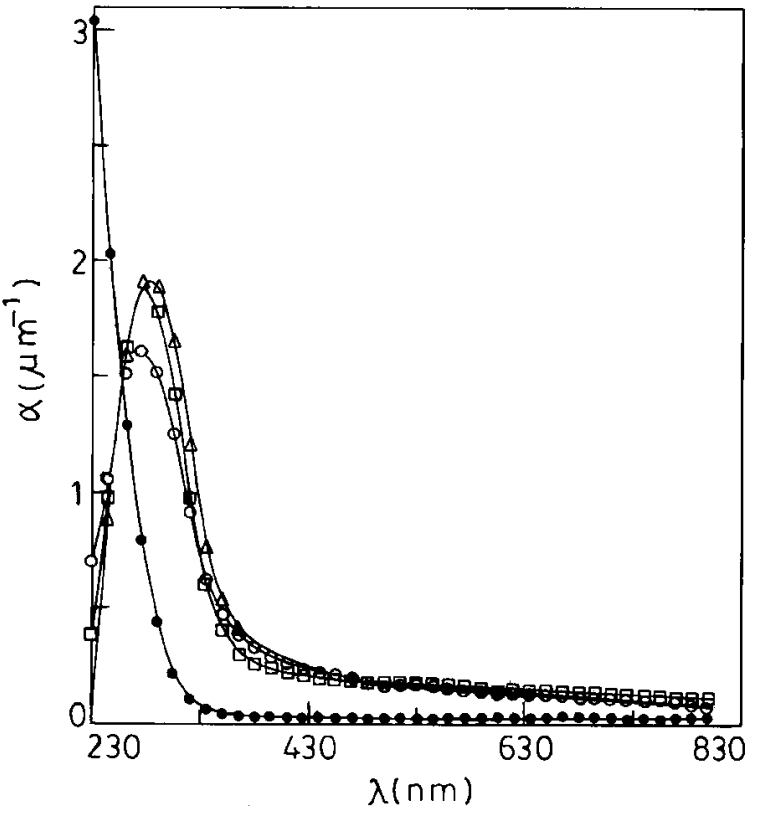

Figure 5 Variation of optical absorption coefficient as a function of wavelength for nanocomposite specimens in the $\mathrm{Fe} /$ $\mathrm{TiO}_{2}$ series: $\bigcirc$, specimen without metal; $\bigcirc$, specimen no. 1; $\square$, specimen no. 2 ; $\triangle$, specimen no. 3 .

also in the specimens containing either nickel or copper. The histograms of particle sizes for all specimens were analysed using Equation [2] as described earlier. Table 4 summarizes the values of mean diameter $\bar{x}$ and geometric standard deviation as extracted by this analysis for all the specimens studied here. It is evident from these results that the particle size of the different metallic species can be increased by raising the reduction temperature and

Table 4. Summary of heat treatment schedule, median diameter and geometric standard deviation for particles in different specimens

\begin{tabular}{|c|c|c|c|c|c|}
\hline \multirow[b]{2}{*}{ Specimen no. } & \multirow{2}{*}{$\begin{array}{c}\text { Nanocomposite } \\
\text { system }\end{array}$} & \multicolumn{2}{|c|}{ Reduction treatment in hydrogen } & \multirow{2}{*}{$\begin{array}{c}\text { Median diameter } \\
\text { (nm) }\end{array}$} & \multirow{2}{*}{$\begin{array}{c}\text { Geometric } \\
\text { standard deviation } \\
\sigma(\mathrm{nm})\end{array}$} \\
\hline & & Temp $\left({ }^{\circ} \mathrm{C}\right)$ & Duration (min) & & \\
\hline 1 & $\mathrm{Fe} / \mathrm{TiO}_{2}$ & 550 & 10 & 12.0 & 1.4 \\
\hline 2 & $\mathrm{Fe} / \mathrm{TiO}_{2}$ & 660 & 10 & 14.3 & 1.6 \\
\hline 3 & $\mathrm{Fe} / \mathrm{TiO}_{2}$ & 675 & 20 & 20.1 & 2.3 \\
\hline 4 & $\mathrm{Ni} / \mathrm{TiO}_{2}$ & 550 & 10 & 12.2 & 1.3 \\
\hline 5 & $\mathrm{Ni} / \mathrm{TiO}_{2}$ & 660 & 10 & 15.7 & 1.2 \\
\hline 6 & $\mathrm{Ni} / \mathrm{TiO}_{2}$ & 675 & 20 & 18.3 & 1.4 \\
\hline 7 & $\mathrm{Cu} / \mathrm{TiO}_{2}$ & 550 & 10 & 6.8 & 1.8 \\
\hline 8 & $\mathrm{Cu} / \mathrm{TiO}_{2}$ & 660 & 10 & 13.1 & 1.9 \\
\hline 9 & $\mathrm{Cu} / \mathrm{TiO}_{2}$ & 675 & 20 & 18.4 & 2.1 \\
\hline
\end{tabular}




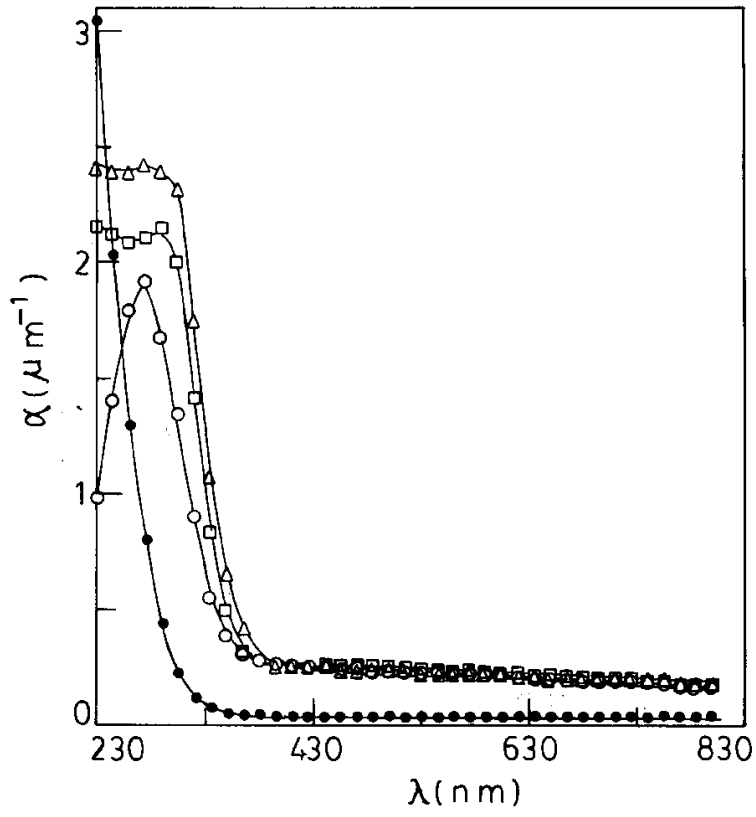

Figure 6 Variation of optical absorption coefficient as a function of wavelength for nanocomposite specimens in the $\mathrm{Ni}$ / $\mathrm{TiO}_{2}$ series: $\bigcirc$, specimen without metal; $\bigcirc$, specimen no. 4; $\square$, specimen no. 5 ; $\triangle$, specimen no. 6 .

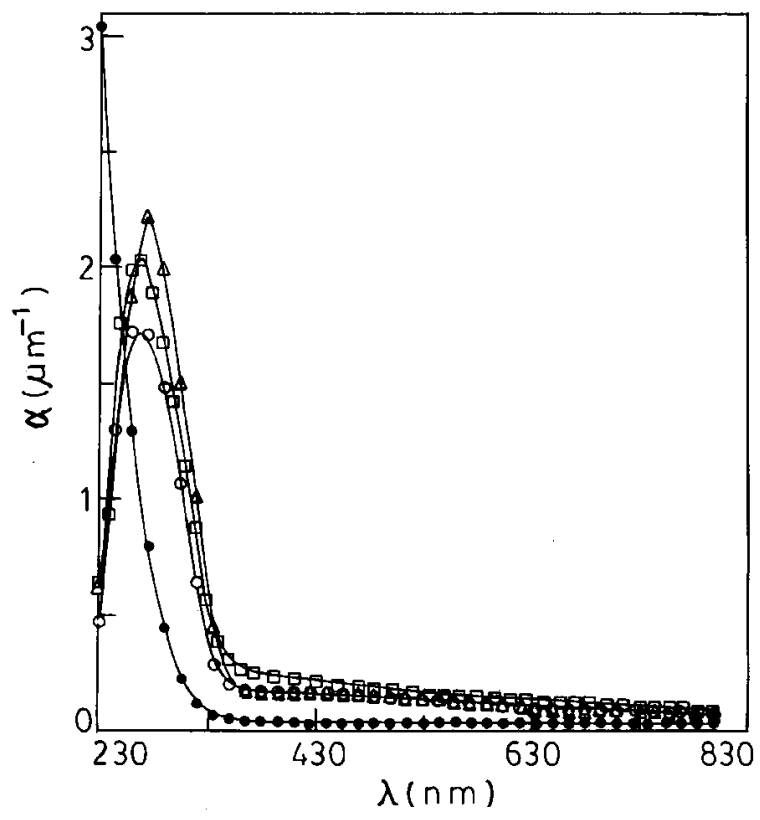

Figure 7 Variation of optical absorption coefficient as a function of wavelength for nanocomposite specimens in the $\mathrm{Cu}$ / $\mathrm{TiO}_{2}$ series: $\bigcirc$, specimen without metal; $\bigcirc$, specimen no. 7; $\square$, specimen no. 8 ; $\triangle$, specimen no. 9 .

Copyright (C) 1999 John Wiley \& Sons, Ltd.

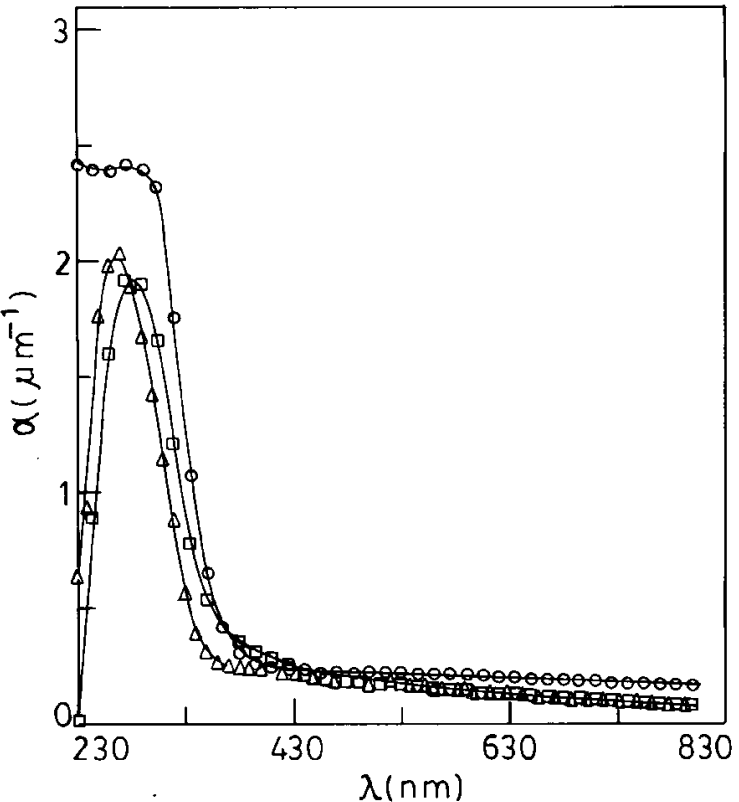

Figure 8 Comparison of optical absorption for nanocomposites containing different metallic species of comparable particle size: $\square$, specimen no. $3 ; \bigcirc$, specimen no. $6 ; \triangle$, specimen no. 9 .

duration of treatment. The particle sizes obtained in the present series of samples range from 6.8 to $18.4 \mathrm{~nm}$. Such an increase is consistent with the diffusion-controlled growth of the metallic phases.

Figure 5 shows the variation of absorption coefficient as a function of wavelength $(\lambda)$ for specimens (1,2 and 3) containing nanoparticles of iron. The curves show an absorption maximum at around $280 \mathrm{~nm}$. The maximum is seen to shift to higher wavelength as the metal particle size is increased. Figures 6 and 7 show the $\alpha$ versus $\lambda$ plots for different specimens in the $\mathrm{Ni} / \mathrm{TiO}_{2}$ and $\mathrm{Cu} / \mathrm{TiO}_{2}$ series respectively. In the former case the maximum is around $300 \mathrm{~nm}$, in the latter case it is found to be around $270 \mathrm{~nm}$. The results are similar to these described in Fig. 5. The absorption mechanism responsible for the behaviour is discussed below. Figure 8 compares the absorption characteristics for nanocomposites containing different metallic phases but having comparable particle diameters. It is evident that the nickel glass composites give the highest absorption whereas those containing copper and iron have absorption values similar to each other. Although no apparent

Appl. Organometal. Chem. 13, 353-360 (1999) 
reason could be adduced for this behaviour, the following discussion and the results obtained by fitting the data to the equations governing effective medium theories suggest that this arises simply because of a higher volume fraction of the metallic phase.

The present series of specimens comprise composites containing nanoparticles of different metallic species dispersed within a glass medium. These are therefore ideal systems for their optical behaviour to be analysed by different effective medium theories developed earlier. We have used Maxwell-Garnett ${ }^{9}$ and Bruggeman ${ }^{10}$ models respectively in the present study.

In the Maxwell-Garnett (MG) model the particles in the medium are assumed to be spherical, having identical diameters and separated from each other so that they scatter light independently. Then the expression for the effective medium dielectric permittivity $\varepsilon^{\mathrm{MG}}$ in this model is given by Equation [3]:

$$
\frac{\bar{\varepsilon}^{\mathrm{MG}}-\varepsilon_{\mathrm{m}}}{\bar{\varepsilon}^{\mathrm{MG}}+2 \varepsilon_{\mathrm{m}}}=f \frac{\varepsilon-\varepsilon_{\mathrm{m}}}{\varepsilon+2 \varepsilon_{\mathrm{m}}}
$$

where $\varepsilon_{\mathrm{m}}$ is the dielectric permittivity of the matrix phase, $f$ is the volume fraction or filling factor of the metal phase in the composite and $\varepsilon$ is the permittivity of individual metal particles.

The Bruggemann (BR) model is based upon the assumption that the particles are embedded in an effective medium in such a way that the average single-site scattering is zero. The effective dielectric permittivity $\varepsilon^{\mathrm{BR}}$ according to this model is given by Equation [4]:

$$
\bar{\varepsilon}^{\mathrm{BR}}=\varepsilon_{\mathrm{m}} \frac{1-f+\frac{1}{3} \sum_{j} f_{j} \beta_{j}}{1-f-\frac{2}{3} \sum_{j} f_{j} \beta_{j}}
$$

where $f_{j}$ is the filling factor of particles with diameters $d_{j}$ such that

$$
\sum_{j} f_{j}=f
$$

where $f$ is the filling factor of the metal phase,

$$
\beta_{j}=\frac{1}{3} \sum_{l=1}^{3} \frac{\varepsilon_{j}-\varepsilon}{\bar{\varepsilon}+L_{l}\left(\varepsilon_{j}-\bar{\varepsilon}^{\mathrm{BR}}\right)}
$$

where $\varepsilon_{j}$ is the size-dependent dielectric permittivity of particles with diameters $d_{j}$ and $L_{l}$ are their depolarization factors.

The size-dependent dielectric permittivity of each of the metallic phases has been calculated

Copyright (C) 1999 John Wiley \& Sons, Ltd.
Table 5. Values of $\omega_{\mathrm{pb}}, \tau_{\mathrm{b}}$ and $\boldsymbol{V}_{\mathrm{Fb}}$ used for calculation of dielectric permittivities for different metallic species

\begin{tabular}{lccc}
\hline $\begin{array}{l}\text { Metallic } \\
\text { phase }\end{array}$ & $\begin{array}{c}\omega_{\mathrm{pb}} \\
\left(10^{15} \mathrm{rad} \mathrm{s}^{-1}\right)\end{array}$ & $\tau_{\mathrm{b}}\left(10^{-15} \mathrm{~s}\right)$ & $\begin{array}{c}V_{\mathrm{Fb}} \\
\left(10^{6}{ }_{\mathrm{m} \mathrm{s}} \mathrm{s}^{-1}\right)\end{array}$ \\
\hline Iron & 6.1 & 2.1 & 2.0 \\
Nickel & 6.5 & 1.9 & 2.0 \\
Copper & 4.5 & 2.0 & 1.6 \\
\hline
\end{tabular}

from the corresponding bulk data using the Equation [7]: ${ }^{12}$

$$
\varepsilon_{j}(\omega)=\varepsilon_{\text {expt }}(\omega)-\varepsilon_{\text {expt }}^{\text {Drude }}(\omega)+\varepsilon_{j}^{\text {Drude }}(\omega)
$$

where $\omega$ is the angular frequency of the radiation concerned,

$$
\begin{aligned}
& \varepsilon_{\text {expt }}^{\text {Drude }}(\omega)=1-\omega_{\mathrm{pb}}^{2} / \omega\left(\omega+i / \tau_{\mathrm{b}}\right) \\
& \varepsilon_{j}^{\text {Drude }}(\omega)=1-\omega_{\mathrm{pd}_{j}}^{2} / \omega\left(\omega-i / \tau_{\mathrm{b}}\right)
\end{aligned}
$$

where $\omega_{\mathrm{pb}}$ and $\tau_{\mathrm{b}}$ are the bulk plasma frequency and mean electron lifetime respectively, $\omega_{\text {pd }_{j}}$ represents the apparent plasma frequency of particle $\mathrm{d}_{j}$, and $\tau_{j}$ is given by

$$
\tau_{j}^{-1}=\tau_{\mathrm{b}}^{-1}+2 V_{\mathrm{Fb}} / d_{j}
$$

where $V_{\mathrm{Fb}}$ is the bulk Fermi velocity of electrons. In the present case we assume $\omega_{\mathrm{pd}_{j}} \approx \omega_{\mathrm{pb}}{ }^{12}$ We have estimated the values of $\omega_{\mathrm{pb}}$ for different metals from the corresponding dielectric permittivity data as a function of frequency, ${ }^{13}$ and $\tau_{\mathrm{b}}$ and $V_{\mathrm{Fb}}$ have been calculated using the free-electron model. ${ }^{14}$ Table 5 summarizes the values of $\omega_{\mathrm{pb}}, \tau_{\mathrm{b}}$ and $V_{\mathrm{Fb}}$ for different metallic species used in our calculation. A value of $\varepsilon_{\mathrm{m}}=17$ has been assumed based on dielectric measurements carried out on gel-derived $\mathrm{TiO}_{2}$ films before any reduction treatment was given. The size-dependent filling factors $f_{j}$ in equations [4] and [5] are estimated from Equation [11]:

$$
f_{j}=\omega_{j} f
$$

where $\omega_{j}$ is the weight factor and is given by

$$
\omega_{j}=d_{j}^{3} n_{j} / \sum d_{j}^{3} n_{j}
$$

$n_{j}$ being the number of particles with diameter $d_{j}$.

The optical absorption coefficient $\alpha$ was calcu-

Appl. Organometal. Chem. 13, 353-360 (1999) 


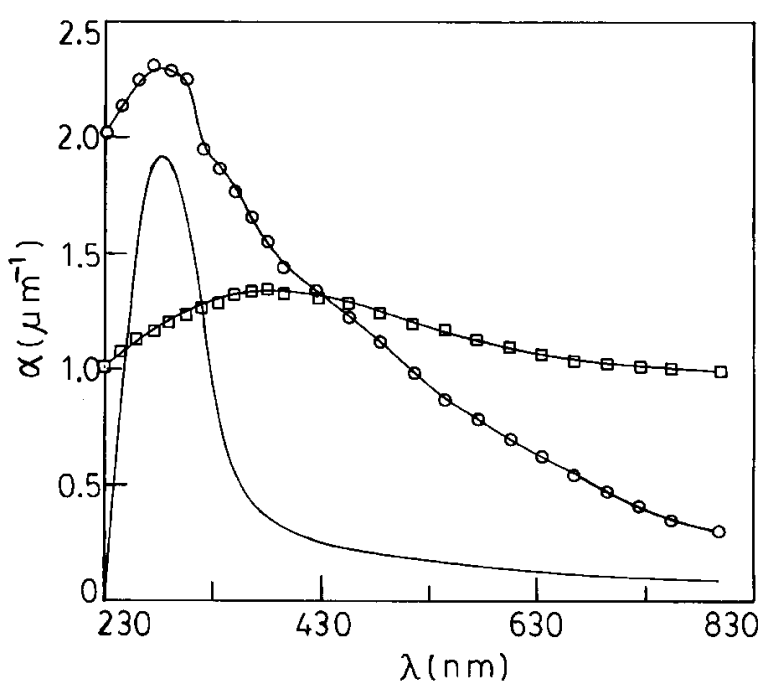

Figure 9 Comparison of experimental with theoretical curves obtained from the MG and BR models for specimen no. 3: experimental; $\bigcirc$, MG model; $\square$, BR model.

lated using Equation [13]

$$
\alpha(\omega)=\left(\frac{\omega}{c}\right)\left(\frac{\bar{\varepsilon}_{2}}{\bar{\varepsilon}_{1}^{1 / 2}}\right)
$$

The experimental data for different specimens were least-squares fitted to theoretical expressions as given above for the two models using $f$ as a

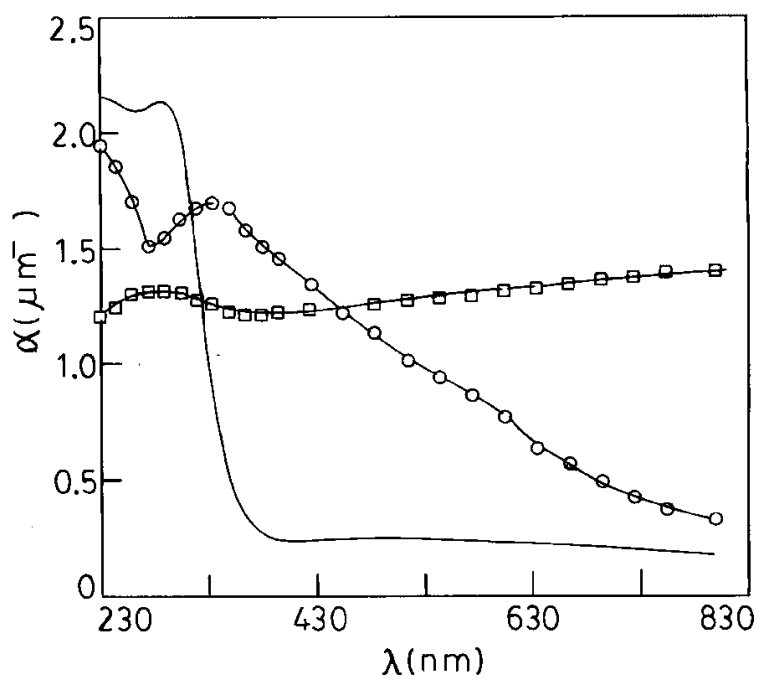

Figure 10 Comparison of experimental with theoretical curves obtained from the MG and BR models for specimen no. 5 ; - , experimental; $\bigcirc$, MG model; $\square$, BR model.

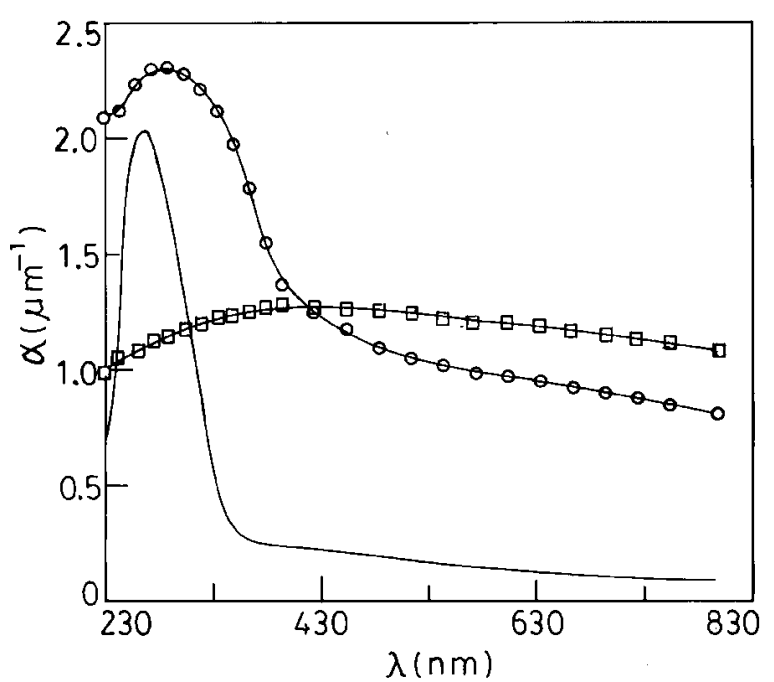

Figure 11 Comparison of experimental with theoretical curves obtained from the MG and BR models for specimen no. 9: - , experimental; $\bigcirc$ the MG model; $\square$, the BR model.

parameter. The value of $f$ could not be estimated from the micrographs because the thicknesses of the samples used for electron microscopy were not known accurately. Figures 9, 10 and 11 show comparisons of experimental results with the theoretical curves for typical specimens in the $\mathrm{Fe} /$ $\mathrm{TiO}_{2}, \mathrm{Ni} / \mathrm{TiO}_{2}$ and $\mathrm{Cu} / \mathrm{TiO}_{2}$ series respectively. It is evident from these Figures that the MaxwellGarnett model gives better qualitative agreement with experiments. This implies that ultrafine metal particles are well separated from each other, thereby satisfying the assumptions made in the Maxwell-Garnett model. The filling factors $f$ as

Table 6. Values of filling factors $f$ for different specimens as estimated by the effective medium theories

\begin{tabular}{lccc}
\hline & & \multicolumn{2}{c}{ Filling factor $f($ vol\%) } \\
\cline { 3 - 4 } Specimen no. & Composite & MG model & BR model \\
\hline 1 & $\mathrm{Fe} / \mathrm{TiO}_{2}$ & 1.7 & 1.2 \\
2 & $\mathrm{Fe} / \mathrm{TiO}_{2}$ & 2.3 & 1.5 \\
3 & $\mathrm{Fe} / \mathrm{TiO}_{2}$ & 2.9 & 2.0 \\
4 & $\mathrm{Ni} / \mathrm{TiO}_{2}$ & 1.9 & 0.8 \\
5 & $\mathrm{Ni} / \mathrm{TiO}_{2}$ & 2.7 & 1.8 \\
6 & $\mathrm{Ni} / \mathrm{TiO}_{2}$ & 3.4 & 2.0 \\
7 & $\mathrm{Cu} / \mathrm{TiO}_{2}$ & 2.2 & 1.9 \\
8 & $\mathrm{Cu} / \mathrm{TiO}_{2}$ & 2.6 & 2.2 \\
9 & $\mathrm{Cu} / \mathrm{TiO}_{2}$ & 3.1 & 2.0 \\
\hline
\end{tabular}

Appl. Organometal. Chem. 13, 353-360 (1999) 
estimated by this fitting procedure are summarized in table 6 for the different specimens. The $f$ values estimated from the MG theory are found to be higher than those calculated from the BR model. It was shown earlier that BR model works better in the case of systems containing clusters of nanoparticles. $^{12}$ Evidently the BR model does not represent adequately the morphological characteristics of the present set of specimens.

In summary, nanocomposite films of thickness $\sim 3 \mu \mathrm{m}$ containing iron, nickel and copper respectively in $\mathrm{TiO}_{2}$ glass matrix were synthesized using a sol-gel technique. The particle sizes were in the range $6.8-20.1 \mathrm{~nm}$, depending on the reduction treatment given to the samples. Detailed optical absorption spectra in the wavelength range 230$830 \mathrm{~nm}$ were recorded. An absorption maximum was observed around $280 \mathrm{~nm}$ in all the specimens. Maxwell-Garnett and Bruggemann models were used to fit the absorption characteristics. The MG theory gives a better qualitative agreement with the experimental results.

Acknowledgements T. K. Kundu acknowledges a Senior Research Fellowship awarded by CSIR, New Delhi. D. Chakravorty acknowledges support from a Special Foreign Currency Programme by the Office of Naval Research, Washington, DC USA, under the Grant no. N 00014-93-1-0040.

\section{REFERENCES}

1. R. Roy, Science 238, 1664 (1987).

2. L. L. Hench, and M. J. R. Wilson, J. Non-Cryst Solids 121, 234 (1990).

3. R. Reisfeld, D. Brusilovsky, M. Eyal, E. Miron, Z. Burshstein, and J. Ivri, Chem. Phys. Lett. 160, 43 (1989).

4. A. Chatterjee, D. Das, D. Chakravorty, and K. Choudhury, Appl. Phys. Lett. 57, 1360 (1990).

5. A. Chatterjee, A. Datta, A. K. Giri, D. Das, and D. Chakravorty, J. Appl. Phys. 72, 3832 (1992).

6. S. Roy, A. Chatterjee, and D. Chakravorty, J. Mater. Res. 8 , 689 (1993).

7. S. Roy, D. Das, D. Chakravorty, and D. C. Agarwal, J. Appl. Phys. 74, 4749 (1993).

8. T. K. Kundu, and D. Chakravorty, J. Mater. Res. 11, 200 (1996).

9. J. C. Maxwell-Garnett, Phil. Trans. R. Soc. 203, 385 (1904).

10. D. A. G. Bruggemann, Ann. Phys. (Leipzig) 24, 636 (1935).

11. A. Kawabata, and R. Kubo, J. Phys. Soc. Jpn. 21, 1765 (1966).

12. C. G. Granqvist, and O. Hunderi, Phys. Rev. 16(8), 3513 (1977).

13. P. B. Johnson, and R. W. Christy, Phys. Rev. B9, 5056 (1974).

14. C. Kittel, Introduction to Solid State Physics, New York Wiley, 1974. 